\title{
Predictive features of HFNC failure in infants with acute bronchiolitis and high-flow nasal cannula
}

\author{
Enrica Mancino ${ }^{1}$, Raffaella Nenna ${ }^{2}$, Domenico Paolo La Regina ${ }^{1}$, Luca Cristiani ${ }^{3}$, Greta Di \\ Mattia $^{1}$, Daniela Pepino ${ }^{1}$, Antonella Frassanito ${ }^{4}$, Laura Petrarca ${ }^{2}$, Luigi Matera ${ }^{1}$, and \\ Fabio Midulla ${ }^{1}$ \\ ${ }^{1}$ University of Rome \\ 2 "Sapienza" University of Rome \\ ${ }^{3}$ Sapienza University of Rome \\ ${ }^{4}$ Policlinico Umberto I
}

July 29, 2020

\begin{abstract}
Introduction Acute bronchiolitis is the most common respiratory illness and the main cause of respiratory failure in infant. Effective therapy is not available. A relatively new, safe and promising method of non-invasive respiratory support is oxygen delivery by high-flow nasal cannula (HFNC), but several questions concerning HFNC clinical practice remain unanswered. Aim The main aim of our study is to analyse the clinical course of infants hospitalized for bronchiolitis who underwent HFNC in order to identify clinical, laboratory or radiological findings that can have an impact on HFNC failure, which is defined as requirement for mechanical ventilation (MV). Methods We conducted a retrospective data analysis of case records of 130 patients less than 12 months hospitalized for bronchiolitis who underwent HFNC and clinical epidemiological laboratory and radiological data were collected. Results Only 11 (8.5\%) out 130 infants required invasive mechanical ventilation for clinical deterioration. Patients who needed to switch from HFNC to MV because of a progressive respiratory failure showed more frequently a complete upper lobe consolidation on CXR (90.9\%) than infants exclusively supported by HFNC (14.9\%). They were younger with a lower admission weight and they had a lower lymphocyte count than patients who underwent HFNC only. Discussion Our study suggests that a complete upper lobe consolidation in young infants is a significant risk factor for HFNC failure. Further studies are needed to understand if an early identification of consolidation following by an adequate follow-up and proper therapeutic strategies may reduce the number of children who require mechanical ventilation.
\end{abstract}

Acute bronchiolitis is the most common respiratory illness and the main cause of respiratory failure in infants, often leading to hospitalization with high healthcare costs [1]. Bronchiolitis is caused by viral infections, particularly by respiratory syncytial virus (RSV), which lead to airway inflammation and obstruction of the lower respiratory tract. Most infants experience mild symptoms similar to those of a common cold; however, symptoms can get worse and become severe and they may develop breathlessness, tachypnea, retractions and hypoxemia with hypercapnia [2]. Effective therapy is not available; treatment is mainly supportive and focuses on management of respiratory distress and hypoxia.

A relatively new, safe and promising method of non-invasive respiratory support is oxygen delivery by highflow nasal cannula (HFNC). HFNC provides a heated and fully humidified mixture of oxygen and air at a pressure slightly more elevated than the inspiratory peak pressure, via a nasal cannula interface. It delivers constant $\mathrm{FiO}_{2}$, improves washout of the nasopharyngeal dead space and mucociliary clearance and it decreases metabolic work related to gas conditioning. Moreover, HFNC creates a low level of positive pharyngeal pressure that might help to reduce inspiratory airway resistance [3]. Several papers demonstrated 
that infants supported by HFNC have a low rate of paediatric intensive care unit (PICU) admission and a reduced need for invasive ventilation. In spite of these beneficial effects, HFNC has not been demonstrated to reduce the length of hospital stay or the duration of oxygen therapy with respect to the oxygen supplementation by nasal prongs $[4,5,6,7]$. In addition, several questions concerning HFNC clinical practice, such as the optimal timing to initiate HFNC and the features that can predict HFNC failure, which is defined as requirement for mechanical ventilation (MV), remain unanswered. Answering to these questions may help in preventing HFNC failure.

Over seven epidemic seasons, we retrospectively analysed the clinical course of infants hospitalized for bronchiolitis who underwent HFNC with the aim of identifying clinical, laboratory or radiological factors that can predict HFNC failure.

\section{MATERIALS AND METHODS}

\section{Study design and population}

Among 824 infants consecutively admitted for bronchiolitis to the Pediatric Emergency Department "Sapienza" University of Rome from 2012 to 2019, we have retrospectively reviewed clinical charts of the 130 infants who received oxygen by HFNC. Bronchiolitis was defined as the first acute lower respiratory tract infection characterized by respiratory distress with tachypnea, cough, retractions and diffuse crackles on auscultation, in full term babies less than 12 months of age [8]. Infants with prematurity, cardiopulmonary disorders, immunodeficiency or congenital anomalies were excluded. Demographical, clinical and laboratory data such as age, gender, breastfeeding, cigarette smoking exposure, body weight, gestational age, days of illness, length of hospital stay and low flow oxygen therapy before HFNC, were systematically collected from clinical charts. On hospital admission and just before starting HFNC, a clinical severity score (from 0 to 8) was assigned to each infant according to respiratory rate, oxygen saturation in room air, presence of retractions and ability to feed [9].

According to our internal protocol, patients underwent HFNC for the following clinical indications: presence of severe retractions and/or nasal flaring associated to respiratory rate higher than 70 breaths per minute and heart rate higher than 150 beats per minute and/or oxygen saturation lower than $92 \%$. HFNC was started with a weight-based gas flow rate, starting with $1 \mathrm{~L} / \mathrm{Kg} / \mathrm{min}$. After 15 minutes, physicians performed a clinical evaluation and if necessary gas flow was titrated up to $2 \mathrm{~L} / \mathrm{Kg}$ and $\mathrm{FiO}_{2}$ adjusted to target $\mathrm{SaO}_{2}$ (over 92\%). A second clinical evaluation was performed after 60 minutes and then every 3 hours over the next 24 hours. In case of progressive respiratory distress and inability to keep $\mathrm{SaO}_{2}$ over $92 \%$ with a FiO2 $60 \%$, patients were admitted to PICU where mechanical ventilation was started.

The study protocol was approved by Policlinico Umberto I ethic committee (Rif. CE 2377/2012).

\section{Radiological findings}

Chest X-ray (CXR) was performed on admission in 105 infants for clinical indications such as when a differential diagnosis was suspected or/and if a clinical deterioration occurred. 31 out of 105 infants $(29.5 \%)$ performed two CXR at least. An experienced paediatric radiologist (D.P.) evaluated each CXR and described the presence of patchy opacities, complete lobar consolidation and air trapping. Radiologist was blinded to infants' conditions and clinical course, in order to avoid potential bias.

\section{Virus detection}

All patients underwent a nasopharyngeal washing (NPW), obtained using $3 \mathrm{ml}$ of sterile saline solution injected into each nostril and collected with a syringe. On NPW, a panel of reverse transcriptase-polymerase chain reaction (RT-PCR) or nested PCR methods was used for the detection of 14 respiratory viruses, including RSV, human rhinovirus (hRV), influenza (Flu) A and B, parainfluenza viruses (PIV) 1-3, adenovirus (ADV), human coronaviruses (hCoVs OC43, 229E, NL-63, HUKI), human metapneumovirus (hMPV) 
and human Bocavirus (hBoV), as described [9]. All specimens were collected within 24 hours of hospital admission.

\section{Statistical analysis}

Statistical analysis was performed using the SPSS Software (version 25.0; SPSS Inc., Chicago, IL). Infants exclusively supported by HFNC and those who required escalation to MV were divided in two groups and demographic, clinical, laboratory and radiological features were compared. Continuous variables values were expressed as mean $\pm \mathrm{SD}$ or median and range and compared using T-student or Mann-Whitney tests. Categorical variables were expressed as number and percentages and compared using chi-square test. $\mathrm{P}<$ 0.05 was considered statistically significant. A multivariate logistic regression analysis was performed to determine factors associated with HFNC failure, which was defined as requirement for MV.

\section{RESULTS}

Among the 824 infants consecutively hospitalized for bronchiolitis in the Paediatric Emergency Department of "Sapienza" University of Rome, 130 (63.8\% males; median age 56.5 days, range 14-319) underwent HFNC and we registered a failure of HFNC in only 11 infants (8.5\%) (Figure 1).

Concerning demographic and clinical characteristics, infants receiving MV were significantly younger and had a lower body weight than infants who underwent HFNC only. Clinical severity score just before starting HFNC was higher in infants who needed MV. The percentage of patients who underwent low flow oxygen therapy before HFNC were significantly higher in infants who needed MV comparing to infants who were successfully treated only with HFNC. Days of disease and clinical severity score at hospital admission did not show statistically significant differences between the two groups (Table 1).

Comparing laboratory and radiological findings, patients who needed MV had a significantly lower lymphocytes count and mean platelet volume/platelet count ratio (PLT/MPV) than infants who underwent HFNC only (Table 2).

Complete lobar consolidation was found in $90.9 \%$ of infants who underwent MV comparing to $14.9 \%$ of patients exclusively supported by HFNC and the upper right lobe consolidation was more frequently detected than the upper left lobe consolidation (Table 2). Analysing infants who required MV, 9 out of 11 patients underwent at least two CXR before MV. In particular, 8 (72.7\%) infants had a gradual development up to a complete upper lobe consolidation, and in one $(9.1 \%)$ no consolidation but severe air trapping and diffuse patchy opacities were found. Moreover, $2(18.2 \%)$ patients showed a complete upper lobe consolidation on first CXR and were directly treated with MV; a second CXR was performed to evaluate infant clinical course.

Analysing viruses' detections, RSV was identified in $81.8 \%$ of patients supported by MV and in $49.6 \%$ of patients underwent HFNC (Table 2).

On multivariate analysis adjusted for age, sex and RSV detection, the significant independent risk factor for escalation to mechanical ventilation was the presence of a complete upper right lobe consolidation (Table 3 ). This result was confirmed when we added the clinical severity score just before starting HFNC and PLT/MPV (data not shown).

\section{DISCUSSION}

In this large single-centre retrospective study, we sought to identify factors that can predict HFNC failure in a homogeneous group of infants hospitalized with severe bronchiolitis. We demonstrated that patients who experienced a failure of HFNC because of a progressive respiratory insufficiency showed more frequently a complete upper lobe consolidation on CXR than infants in whom HFNC allowed to overcome respiratory distress. They were younger and had a lower PLT/MPV and lymphocyte count than patients who underwent HFNC only.

In this study, the main feature associated to HFNC failure was the presence of a complete upper lobe consolidation that affected $90.9 \%$ of patients requiring escalation to MV. These infants were also younger 
and the underdeveloped collateral ventilation together with a particularly acute angle of the right upper lobe bronchus may predispose young infants to atelectasis [10]. Strong evidence has demonstrated that age less than 3 months and low body weigh are significant severe bronchiolitis risk factors [11, 12]. In fact, anatomic factors play a key role: the neonates and young infants' airways are small and more easily get obstructed, causing respiratory distress and therefore the recourse to mechanical ventilation. In case of a complete lobar consolidation with airflow obstruction, airways resistance increases and this aspect as well as the decreased lung compliance may contribute to respiratory distress [13]. Providing low levels of positive airway pressure, HFNC could be able to prevent upper airways collapse and to allow alveolar recruitment. Meanwhile, positive end-expiratory pressure (PEEP) generated by invasive mechanical ventilation may also help to overcome airway resistance and atelectasis. Presumably, patients requiring escalation to MV had a severe ventilation/perfusion (V/Q) mismatch of an extent, which HFNC was not enough to overcome hypoxia and respiratory distress.

Considering the timing of the lung involvement, among patients who needed MV the complete upper lobe consolidation was not sudden: $72.7 \%$ of them had a gradual development of atelectasis on CXR (Figure 2 ). For this reason, it could be useful an early identification of consolidation and a follow up by a simple, non-invasive and easy to perform technique, such as for example lung ultrasound $[14,15]$. This remarkable evidence is also supported by data from the four patients that arrived over the same seven epidemics in our Emergency Department in such severe conditions that underwent MV directly and all had an upper lobe consolidation on CXR (data not shown). An early identification of these patients may allow physicians to consider a preventive treatment and to monitor closely them with a proper recognition of deterioration.

Analysing possible treatments of this condition, ample evidence reports that the use of recombinant human DNase in the management of severe RSV bronchiolitis improves clinical conditions and chest radiograph and leads to resolution of atelectasis [16,17]. Recent reports described that neutrophil, massively recruited in RSV bronchiolitis, release neutrophil extracellular traps (NETs) in the extracellular space in response to several pathogens. NET entraps and facilitates the killing of microorganisms but excessive NET release has detrimental effects, causing lung injury [18]. NETs could be degraded by recombinant human DNase (rhDNase), pointing out that nebulised rhDNase might have a fundamental role in severe bronchiolitis complicated by atelectasis [19]. Supporting these data, we report a clinical case of one of patients enrolled suffering from severe bronchiolitis and supported by HFNC. CXR showed a segmental upper lobe consolidation, clearly improved after nebulised rhDNAse (Figure 3).

Another possible therapeutic option comes from several papers evaluating the clinical utility of chest physiotherapy in infants with bronchiolitis even if results are conflicting. A recent review shows that chest physiotherapy does not influence clinical course in hospitalised infants with acute bronchiolitis [20]. Nevertheless, since airway clearance techniques improve mobilization and transport of secretions reducing airway obstructions by mucus plugs [21], chest physiotherapy could be implemented, particularly in selected infants with bronchiolitis and CXR consolidation.

The analysis of the clinical severity score at HFNC positioning showed that infants who required escalation to MV presented a higher disease severity with an increased respiratory effort, while no differences in clinical severity score at hospital admission or in days of disease between the two groups were found. Moreover, HFNC failure was noticed mostly in infants supported by standard oxygen therapy before starting HFNC. These findings suggest the importance of not postponing the use of HFNC; infants with first signs of respiratory distress could benefit from an early use of HFNC.

When we evaluated laboratory predictors of HFNC failure, we demonstrated a decreased number of peripheral blood lymphocytes and a low PLT/MPV ratio in infants with higher disease severity and escalation to mechanical ventilation. Recent reports showed that PLT/MPV ratio is a promising biomarker helping to discriminate between sepsis and viral disease [22]; moreover, lymphocytes depletion is associated to more severe forms of bronchiolitis [23].

In conclusion, our main finding is that a complete upper lobe consolidation in young infants is a significant 
risk factor for HFNC failure and for the recourse to mechanical ventilation. Further studies are needed to understand if an early identification of consolidation following by an adequate follow-up and by proper therapeutic strategies may further reduce the number of children who require mechanical ventilation.

\section{Figures}

Figure 1. Study design and study population.

Figure 2. Chest X-ray at hospital admission (A) and after clinical worsening (B), showing gradual development of complete upper right lobe consolidation in one infant hospitalized for bronchiolitis.

Figure 3. Segmental consolidation of the upper right lobe in infant with severe bronchiolitis who needed HFNC only (A). Improved aeration after 3 days of nebulized recombinant human deoxyribonuclease (B).

\section{References}

1. H. Cody Meissner, M.D. Viral Bronchiolitis in Children. N Engl J Med 2016;374:62-72.

2. Ralston SL, Lieberthal AS, Meissner HC, et al. Clinical practice guideline: the diagnosis, management, and prevention of bronchiolitis. American Academy of Pediatrics. 2014; 134:e1474-e1502.

3. Slain KN, Shein SL, Rotta AT. The use of high-flow nasal cannula in the pediatric emergency department. J Pediatr. 2017; 93:36-45.

4. Franklin D, Babl FE, Schlapbach LJ et al. A randomized trial of high-flow oxygen therapy in infants with bronchiolitis. N Engl J Med. 2018;378:1121-1131.

5. Kepreotes E, Whitehead B, Attia J et al. High-flow warm humidified oxygen versus standard lowflow nasal cannula oxygen for moderate bronchiolitis (HFWHO RCT): an open, phase 4, randomised controlled trial. Lancet. 2017; 389:930-939.

6. Hilliard TN, Archer N, Laura $\mathrm{H}$ et al. Pilot study of vapotherm oxygen delivery in moderately severe bronchiolitis. Arch Dis Child. 2012; 97:182-183.

7. Ergul AB, Calıskan E, Samsa H, et al. Using a high-flownasal cannula provides superior results to OxyMask delivery in moderate to severe bronchiolitis: a randomized controlled study. Eur J Pediatr.2018; 177:1299-1307.

8. Petrarca L, Nenna R, Frassanito A, et al. Bronchiolitis under 6 months of age: time for changing definition? European Respiratory Journal. 2018, 52 (suppl 62) PA4641; DOI: 10.1183/13993003.

9. Midulla F, Scagnolari C, Bonci E, et al. Respiratory syncytial virus, human bocavirus and rhinovirus bronchiolitis in infants. Arch Dis Child. 2010; 95: 35-41.

10. Terry PB, Traystman RJ. The clinical significance of collateral ventilation. Ann Am Thorac Soc. 2016;13(12):2251-2267.

11. Papoff P, Moretti C, Cangiano C, et al. Incidence and Predisposing Factors for Severe Disease in Previously Healthy Term Infants Experiencing Their First Episode of Bronchiolitis. Acta Paediatr. 2011; 100(7):e17-23.

12. Sinha I, McBride A, Smith R, et al. CPAP and High-Flow Nasal Cannula Oxygen in Bronchiolitis. Chest. 2015;148(3):810-823.

13. Reuter S, Moser C, Baack M. Respiratory distress in the newborn. Pediatr Rev. 2014;35(10):417-429.

14. Basile V, Di Mauro A, Scalini E, et al. Lung ultrasound: a useful tool in diagnosis and management of bronchiolitis. BMC Pediatr. 2015;15:63.

15. Bloise S, La Regina D, Pepino D, et al. Lung ultrasound compared to chest X-ray for the diagnosis of CAP in children. Pediatrics International 2020, in press.

16. Merkus $\mathrm{P}$, de Hoog $\mathrm{M}$, van Gent $\mathrm{R}$, et al. DNase treatment for atelectasis in infants with severe respiratory syncytial virus bronchiolitis. Eur Respir J. 2001; 18: 734-737

17. Nenna R, Tromba V, Berardi R, et al. Recombinant Human Deoxyribonuclease Treatment in Hospital Management of Infants with Moderate-Severe Bronchiolitis. European Journal of Inflammation, 2009;169-174.

18. Porto BN, Stein RT. Neutrophil Extracellular Traps in Pulmonary Diseases: Too Much of a Good Thing? Front. Immunol. 2016; 7:311. 
19. Enriquez A, Chu IW, Mellis C, et al. Nebulised deoxyribonuclease for viral bronchiolitis in children younger than 24 months. Cochrane Database of Systematic Reviews 2012, Issue 11. Art. No.: CD008395.

20. Roqué i Figuls M, Giné-Garriga M, Granados Rugeles C, et al. Chest physiotherapy for acute bronchiolitis in paediatric patients between 0 and 24 months old. Cochrane Database Syst Rev. 2012;(2):CD004873.

21. Oberwaldner B. Physiotherapy for airway clearance in paediatrics. European Respiratory Journal. 2000; 15 (1) 196-204.

22. Ates S, Oksuz H, Dogu B, et al. Can mean platelet volume and mean platelet volume/platelet count ratio be used as a diagnostic marker for sepsis and systemic inflammatory response syndrome? Saudi Med J. 2015;36(10):1186-1190.

23. Russell CD, Unger SA, Walton M, Schwarze J. The Human Immune Response to Respiratory Syncytial Virus Infection. Clin Microbiol Rev. 2017; 30(2):481-502.

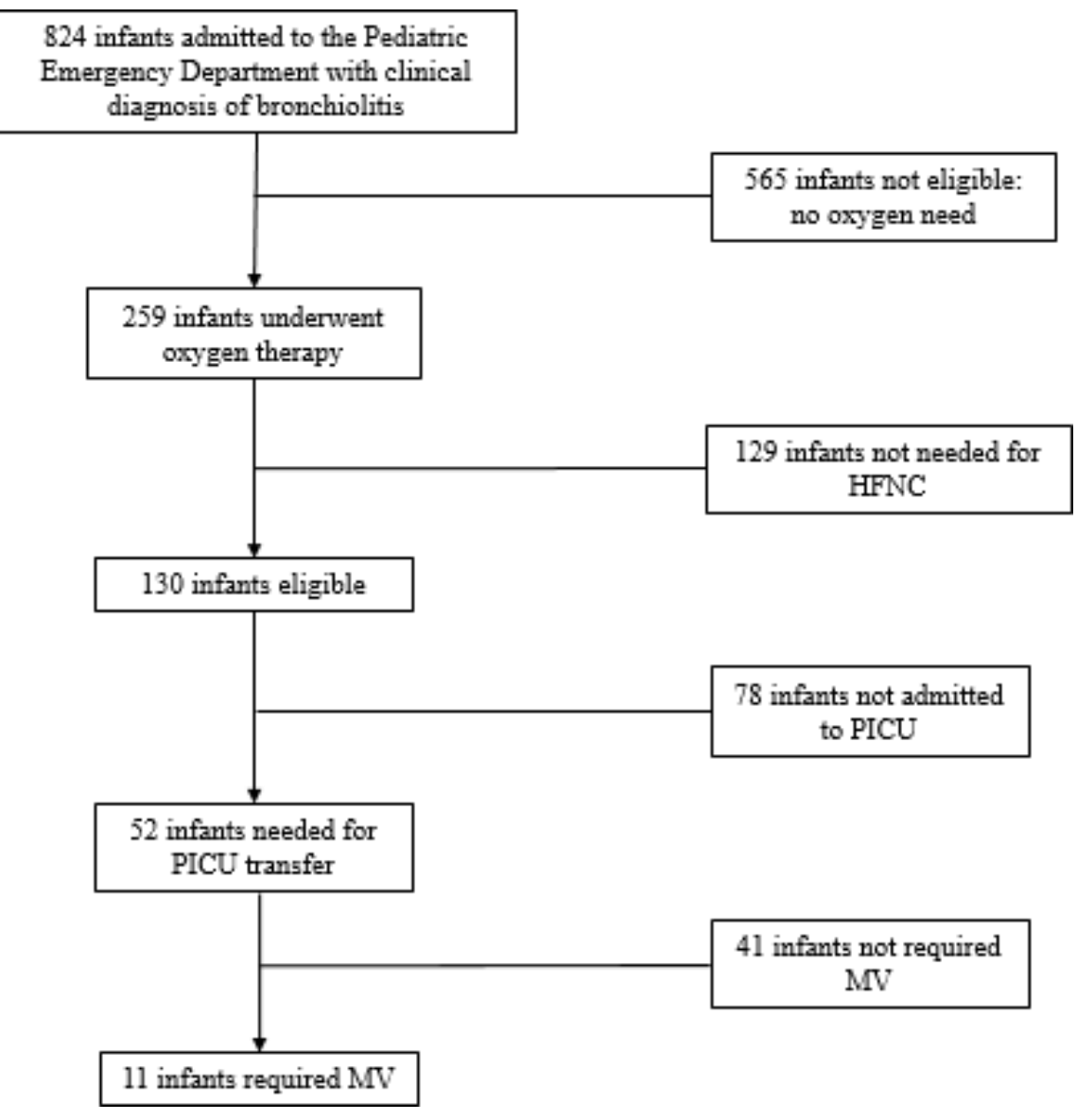



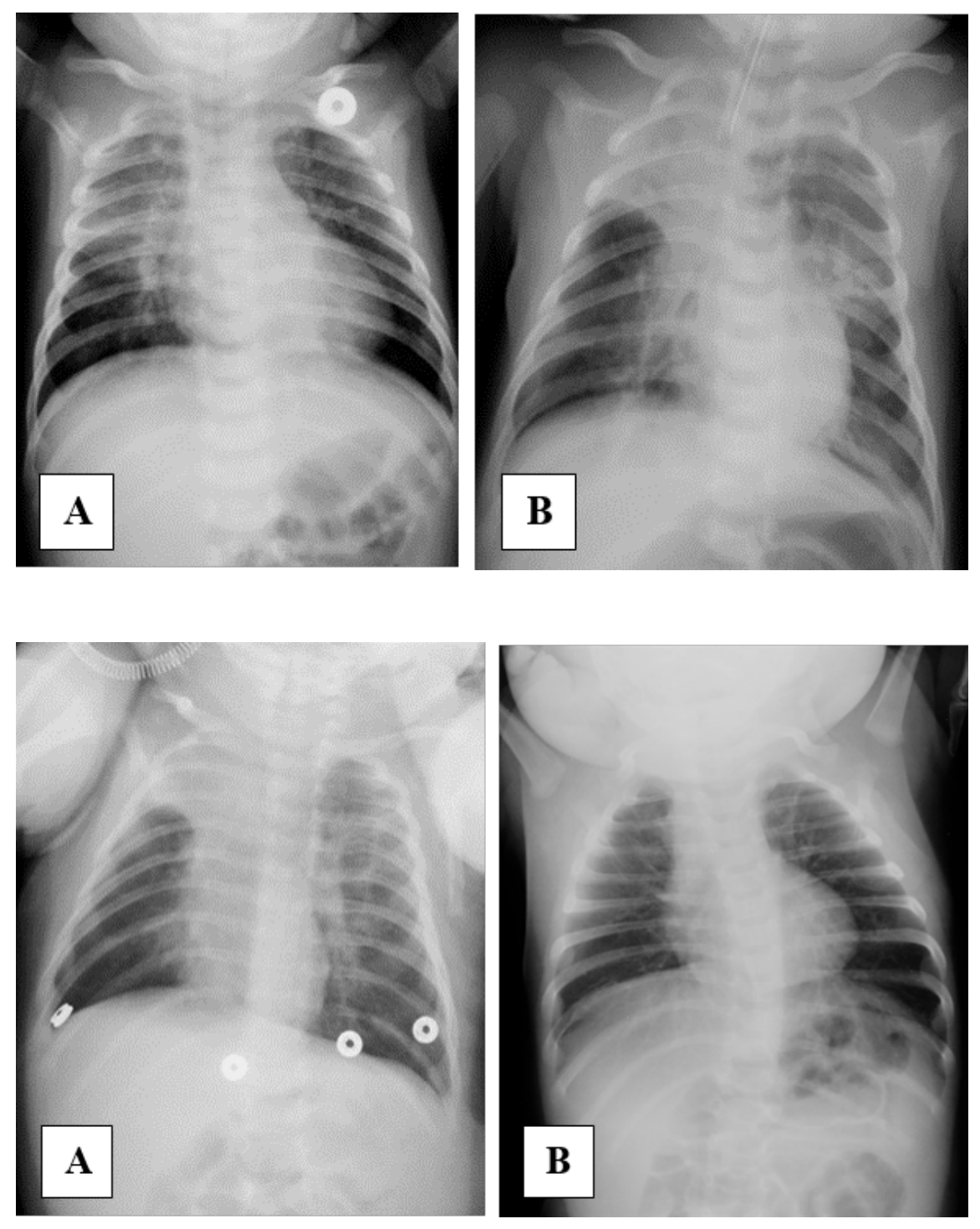

Hosted file

Tables.docx available at https://authorea.com/users/347244/articles/473009-predictivefeatures-of-hfnc-failure-in-infants-with-acute-bronchiolitis-and-high-flow-nasal-cannula 